\title{
Assessing Quality of Pharmacist-Led Education for Patients with COPD Using the Lung Information Needs Questionnaire: A Pilot Study
}

Teri Barnett, PharmD ${ }^{1}$; Anastasia Jenkins, PharmD²; Alicia Bouldin, PhD²; Melvin Crumby, PharmD ${ }^{1}$; Alice K. Morgan, PharmD Michael L. Warren, PharmD ${ }^{2}$

${ }^{1}$ Baptist Memorial Hospital-North Mississippi; ${ }^{2}$ University of Mississippi School of Pharmacy

\begin{abstract}
Background: Transitioning a patient from the hospital to home is an area of vulnerability for patients with Chronic Obstructive Pulmonary Disease (COPD). Patients with COPD frequently readmit as they often do not understand their disease state, medications or when to seek medical attention. Objectives: The objective of this study is to determine the impact that pharmacist-led education has on a patient's understanding of their disease state by assessing the results of the Lung Information Needs Questionnaire (LINQ). Methods: This study uses a quasi-experimental design to formally assess pharmacist-led education provided to patients with COPD using the LINQ. The LINQ was used to assess knowledge of the disease state and medications before and after receiving education on disease state management, smoking cessation and proper medication use. Results: A total of 17 patients completed the LINQ. The survey results showed a statistically significant improvement in patient understanding in 4 of the 6 targeted areas. Conclusion: Results from the formal assessment using the LINQ suggest that pharmacist-lead education for COPD patients is beneficial and reliable. This study presents a continued need for patient education and research in this high-risk patient population.
\end{abstract}

Key Words: Patient education, LINQ, Chronic Obstructive Pulmonary Disease, Transitions of Care, Smoking Cessation

\section{Introduction}

Chronic Obstructive Pulmonary Disease (COPD) is a progressive respiratory illness that contributes considerably to morbidity and mortality as the third leading cause of death in the United States. As the disease progresses, patients will experience increased dyspnea, sputum production, coughing, inability to perform activities of daily living, and an overall decline in mental and physical wellbeing. [1]

Transitioning a patient home after discharge has historically been an area of vulnerability for patients with Chronic Obstructive Pulmonary Disease (COPD).[1] Studies have shown that patients with COPD often do not understand their disease state, how to properly use their medications or when to seek treatment during an exacerbation.[2-4] Qualitative research has described the need for pharmacist integration into transitions of care (TOC) teams to prevent medication-related problems and identify components that contribute to 30-day readmissions. However, more studies are needed to determine the correlation of pharmacist involvement on TOC teams and reduction in readmission rates. $[5,6]$

Baptist Memorial Hospital - North Mississippi (BMH-NM), a 217-bed community hospital in Northeast Mississippi, introduced a pharmacist-led COPD transitions service in December 2017. The pharmacist-led COPD service protocol provided physicians with the option to consult pharmacy to perform disease state management services for patients

Corresponding author: Teri Barnett, PharmD

Baptist Memorial Hospital-North Mississippi

Email: teribarnett12@gmail.com admitted with a COPD exacerbation. Following the initiation of the service, the Lung Information Needs Questionnaire (LINQ) was identified as a useful instrument to assess the benefit of the education pharmacists were providing.

The LINQ is an instrument used to assess the education needs of patients with COPD. It consists of 16 items and 6 domains. [7, 8] The six domains each have subscale scores: disease state knowledge (0-4), medication knowledge (0-5), selfmanagement ability (0-6), smoking status (0-3), knowledge of exercise requirements (0-5) as well as knowledge of an appropriate diet (0-2). Higher scores in each domain indicate a greater need for education in that area per respondent. The total summed score has a range from 0 to 25 , with a score of 0 indicating complete understanding, and a score of 25 indicating the need for education in all areas. [7-9]

Upon consultation, a pharmacist would provide thorough disease state education and medication management in accordance with the pharmacist-led COPD service protocol. Additionally, in order to assess the benefit and reliability of the education being provided by pharmacists, the Lung Information Needs Questionnaire (LINQ) would be given to a group of patients prior to and after education.

The primary objective of this study was to determine the impact that pharmacist-led education has on a patient's understanding of their disease state by assessing the results of the Lung Information Needs Questionnaire prior to and following patient education sessions. 


\section{Methods}

This study was approved by the Institutional Review Board of Baptist Memorial Healthcare Corporation (BMHCC) to be conducted at BMH-NM. Patients included in the study were over the age of 18 years, were admitted with a COPD exacerbation and for whom pharmacy was consulted for COPD education and management. Patients were excluded if they did not speak English or were unable to understand the contents of the survey.

The pharmacist-led COPD transitions service began in December 2017 with pharmacists completing consultations per standard protocol. The pharmacy department would be consulted by a physician to initiate the COPD service. After consultation, the pharmacist would perform disease state and smoking cessation education during the initial visit. Then the pharmacist would confer with the physician to determine the patient's needs inpatient and upon discharge. Finally, prior to discharge, the pharmacist would return to educate on proper inhaler technique, ensure optimization of home COPD medications, and answer all patient questions.

IRB approval for the administration of the LINQ survey and chart review was obtained in February of 2018 and all patients seen by the pharmacy department from that point forward were invited to participate in the survey. With each consult initiation, the patient was given the opportunity to receive education with or without completing the LINQ. If the patient opted to participate in the survey, it was administered either orally by the pharmacist or completed as a written assessment by the patient. The baseline data collected included patient name, age, gender, smoking history, home/inpatient medication regimen and primary care provider and/or pulmonologist.

After completion of the survey, the pharmacist performed disease state education, smoking cessation education, demonstration of inhaler technique, assessment and optimization of home and hospital COPD treatment regimens and medication procurement (if financial need was evident). Disease state and smoking cessation education and therapy optimization most often occurred during the initial visit immediately after completion of the LINQ, while demonstration of inhaler technique and acquisition of medication transpired closer to the patient's discharge. The pharmacist performed a follow-up call for LINQ participants 48-72 hours after discharge. During the follow-up call, the pharmacist repeated the questions from the LINQ survey and the responses were compared to the pre-education responses. The pharmacist also verified that the patient was able to acquire all discharge medications and was aware of their follow-up visit with their provider. Finally, the pharmacist reviewed the patient's wellbeing and ability to self-manage his or her disease state, including key points regarding when to seek help in an exacerbation.

\section{Results}

A total of 18 patients were available and agreed to participate in the LINQ. One of the 18 surveyed patients was not able to comprehend the contents of the questionnaire and was excluded from the LINQ analysis. Thus, the analysis for the LINQ was based on 17 patients (female $=10 ; 59 \%$ ). The average age was 73 years (range $=51-89$ years).

The majority of LINQ participants (88\%) were either smokers or former smokers, and all patients were determined to have severe COPD (severe COPD was categorized as any patient who required oxygen, had documented spirometry $<50 \%$, or had greater than 2 hospital admissions for COPD in one year). Only $35 \%$ of participants had been seen by a pulmonologist at any point, during a hospitalization or as an outpatient, and all participants were either Medicaid, Medicare or both Medicaid/Medicare patients. Characteristics for patients who completed both pre- and post-education LINQ surveys are shown in Table I.

\begin{tabular}{|c|c|}
\hline Gender & $\begin{array}{l}59 \% \text { Female }(n=10) \\
41 \% \text { Male }(n=7)\end{array}$ \\
\hline Smoking Status & $\begin{array}{l}53 \% \text { Former smoker }(n=9) \\
35 \% \text { Current smoker }(n=6) \\
12 \% \text { Never smoker }(n=2)\end{array}$ \\
\hline $\begin{array}{l}\text { COPD stage (mild, moderate, } \\
\text { severe) }\end{array}$ & $100 \%$ severe $(n=17)$ \\
\hline Admission History in one year & $\begin{array}{l}47 \% \text { had } 2-3 \text { admissions }(n=8) \\
53 \% \text { had }>3 \text { admissions }(n=9)\end{array}$ \\
\hline Age of Diagnosis & $\begin{array}{l}24 \% 50-64 \text { years }(n=4) \\
47 \% 64-74 \text { years }(n=8) \\
29 \%>74 \text { years }(n=5)\end{array}$ \\
\hline Pulmonologist/PCP & $\begin{array}{l}35 \% \text { had pulmonologist }(n=6) \\
82 \% \text { had PCP }(n=11)\end{array}$ \\
\hline Insurance Status & $\begin{array}{l}53 \% \text { Medicaid/Medicare }(n=9) \\
41 \% \text { Medicare alone }(n=7) \\
6 \% \text { Medicaid alone }(n=1)\end{array}$ \\
\hline
\end{tabular}

Table II presents information gathered about participants' medication use upon admission and at discharge. The majority of patients (88\%, $n=14)$ had identified medication problems (unaffordability, inappropriate medications, and noncompliance) upon admission. Pharmacists provided recommendations to physicians where appropriate $(n=11)$, and $82 \%$ of these recommendations were accepted. Of the 17 patients surveyed, $2(11.8 \%)$ patients were readmitted, one with a non - COPD related problem. 


\begin{tabular}{|l|c|}
\hline Table II: Population Data Identifying Medication Use (n=17) \\
\hline Medication Problems & $88 \%$ med problems \\
Identified & $\bullet \quad 20 \%$ unaffordability \\
& $-\quad 67 \%$ inappropriate meds \\
& $\bullet \quad 40 \%$ noncompliance \\
\hline $\begin{array}{l}\text { Recommendation } \\
\text { Accepted }\end{array}$ & $\begin{array}{l}82 \% \text { of prescribers accepted } \\
\text { recommendation from pharmacist }\end{array}$ \\
\hline $\begin{array}{l}\text { Discharge meds } \\
\text { prescribed at discharge }\end{array}$ & $\begin{array}{l}88 \% \text { of patients given a prescription at } \\
\text { discharge }\end{array}$ \\
\hline $\begin{array}{l}\text { Readmitted within } \mathbf{3 0} \\
\text { days }\end{array}$ & 2 readmissions (11.76\%) \\
\hline
\end{tabular}

Table III depicts information on the outcomes of the LINQ; all comparisons are documented here. A Wilcoxon signed rank test was used to compare LINQ scores prior to education (pretest) and after discharge (posttest). Baseline total and individual domain scores were consistently higher in all participants with post-education scores being significantly improved across all domains, with the exception of diet and smoking habits. The medication education domain improved the most $(p=0.002)$, followed by self-management $(p=0.002)$. Baseline scores for self-management were the highest among the 6 domains, meaning that patients often reported that they had not been told when to call an ambulance versus when to self-treat or seek attention from their primary care physician. After education by a pharmacist, this figure significantly improved.

Medication management was the area of most improvement. Prior to institutional pharmacist-led education, many patients stated they had never been instructed on how to properly use their inhalers or why they were a necessity. This area also showed a statistically significant change after education and inhaler technique was provided $(p=0.002)$. Each consult involving the LINQ was recorded for time, and pharmacist intervention took an average of 30 minutes (range of $10-90$ minutes) with each consult (LINQ, education, and medication management) during this pilot period.

\begin{tabular}{|l|l|l|l|}
\hline \multicolumn{4}{|l|}{ Table III: LINQ Results Using a Paired T-test (n= 17) } \\
\hline Study Parameter & $\begin{array}{l}\text { Mean } \\
\text { Pre-Test } \\
(\mathrm{n}=17)\end{array}$ & $\begin{array}{l}\text { Mean } \\
\text { Post } \\
\text { Test } \\
(\mathrm{n}=17)\end{array}$ & $\begin{array}{l}\text { P-value } \\
\text { (alpha }= \\
0.05) \\
(\mathrm{n}=17)\end{array}$ \\
\hline Total LINQ score (0-25*) & $12(5-17)$ & $\begin{array}{l}4.77 \\
(0-15)\end{array}$ & $<0.001$ \\
\hline Disease State Education (0-4*) & 2.11 & 0.94 & 0.002 \\
\hline Medication Education (0-5*) & 2.11 & 0.764 & 0.002 \\
\hline Self-management Education (0-6*) & 3.82 & 1.29 & 0.002 \\
\hline Exercise (0-5*) & 2.88 & 1.29 & 0.003 \\
\hline Diet (0-2*) & 0.71 & 0.35 & 0.063 \\
\hline
\end{tabular}

*Higher numbers indicate a greater need for education.

\section{Discussion}

The results associated with pharmacist intervention suggest a trend toward positive outcomes and were documented by the LINQ survey results (see Table III).

The LINQ survey detected areas of information needs and allowed pharmacists to provide targeted education to patients in our population. All 6 domain scores improved, with 4 domains being statistically significant. The smoking domain was a baseline measurement and therefore did not qualify for statistical evaluation. Diet was also an area of least impact as patients expressed awareness of diet needs, but lacked motivation for change.

This study revealed areas of weakness in standard education provided to patients with COPD who have frequent readmissions. Medication education was the most well received/appreciated education provided by pharmacists as was commonly voiced by patients at follow-up. Selfmanagement areas were most improved as patients were given a documented action plan for how to appropriately manage an acute exacerbation. While education was essential in improving patient outcomes, pharmacist intervention in ensuring discharge medications included both rescue and maintenance inhalers was impactful and accepted by physicians $82 \%$ of the time.

There were a number of limitations observed during this study period. One of the most significant challenges faced during this pilot was the lack of additional staffing. All participants were seen and followed by one pharmacist who incorporated the service into her daily workflow. This created difficulty with prioritization of patients and the ability to include more patients into the study. All readmissions may not have been captured, as data were only available to be collected on those who were re-hospitalized at BMHCC affiliates.

IRB approval for the incorporation of the LINQ was not obtained until February of 2018; therefore, only 17 patients were included in the study. The information from the LINQ portion of the study was presented at a conference, all data was to be included by April of 2018, which determined the stop date for administering the LINQ to patients. The LINQ was very time consuming and proved to be an unlikely addition to regular practice in an inpatient setting. However, the information collected on this small number of patients gave us great insight into the typical education needs of our patient population. The results also suggest that the education provided by a pharmacist was well received and appeared to have a useful and significant impact on patient understanding of their disease state and management.

The exclusion criteria of determining a patient's ability to understand was decided by the researcher who had direct contact with the patients. Patients were deemed "unable to understand" if they were asked a question and did not give a 
formed answer, did not answer at all, or regularly stated "I don't know". Therefore, this mode of patient exclusion could have introduced selection bias. A potential confounder on 30-day readmissions in this study was the involvement of a pulmonologist in some patients. While neither of the 2 readmits in this study were treated by pulmonology, it is difficult to conclude that pulmonologist involvement had a direct relationship in readmission status.

Time required per consult, lack of staff participation, and transitioning control of this service to a new staff member all contributed to the lack of data collected beyond April of 2018. This pilot study was originally a resident project that continues to be improved at this facility to incorporate all staff. The data showed positive trends for justification of this service and therefore remains in place at this facility. Improvements continue to be made to this pharmacy service for maximum benefit within a more manageable time commitment.

\section{Conclusion}

In spite of the limitations of this study, the results from the LINQ data showed a clear positive trend of patient improvement in disease state and medication understanding after a pharmacist provided education and medication recommendations. As time and staffing was a problem identified in this pilot, changes are being made to ensure a streamlined approach to providing this service using all clinical and resident pharmacy staff at this institution.

Use of the LINQ allowed for identification of specific areas of education need, thus allowing for a more streamlined approach to patient education among all patients pharmacy was consulted to manage in this study and for future patients. Pharmacist involvement with patient care transitions using targeted education techniques appeared to improve patient understanding and demonstrated trends toward decreased 30day readmission rates. This pilot study showed a need for continued research in order to adjust for seasonal readmission confounders, as well as expand the service to incorporate all pharmacy team members. [10-12]

Conflicts of Interest: We declare no conflicts of interest or financial interests that the authors or members of their immediate families have in any product or service discussed in the manuscript, including grants (pending or received), employment, gifts, stock holdings or options, honoraria, consultancies, expert testimony, patents and royalties.

Treatment of Human Subjects: IRB review/approval required and obtained through Baptist Memorial Healthcare Corporation

\section{References}

1. Centers for Disease Control and Prevention (CDC). National Center for Health Statistics. Deaths: Final data for 2012. Natl Vital Stat Rep 2017; 60(03):1-117.

2. Krishnan JA, Gussin HA, Prieto-Centurion V, Sullivan JL, Zaidi F, Thomashow BM. National COPD Readmissions Summit: Integrating COPD into patientcentered hospital readmissions reduction programs. $J$ COPD F 2015; 2(1): 70-80.

3. Fidahussein S, Croghan I, et al. Posthospital follow-up visits and 30-day readmission rates in chronic obstructive pulmonary disease. Dovepress 2014 May:7;105-112.

4. Vogelmeier CF, Criner GJ, Martinez FJ, Anzueto A, Bar nes PJ, Bourbeau J, Celli BR, Chen R, Decramer M, Fab bri LM, et al. Global strategy for the diagnosis, management, and prevention of chronic obstructive lung disease 2017 report: GOLD executive summary. Am J Respir Crit Care Med 2017;195:557582.

5. Rodrigues $C$, Harrington A, et al. Effect of pharmacysupported transition-of-care interventions on 30-day readmissions: A systematic review and meta-analysis. Ann Pharmacother 2017;51(10)86-889.

6. Bradley EH, Curry L, Horwitz LI, et al. Contemporary evidence about hospital strategies for reducing 30day readmissions: a national study. J Am Coll Cardiol 2012;60:607-614.

7. Lung information needs questionnaire. Plymouth University, 2011. Available from: http://www.linq.org.uk/.

8. Hyland ME, Jones RCM, Hanney KE. The lung information needs questionnaire: development, preliminary validation and findings. Respir Med 2006;100:1807e16.

9. Jones R, Wang X, et al. Educational impact of pulmonary rehabilitation: Lung Information Needs Questionnaire. Respiratory Medicine 2008;102:14391445.

10. Simmering JE, Polgreen LA, Comellas AP, Cavanaugh JE, Polgreen PM. Identifying patients with COPD at high risk of readmission. Chronic Obstr Pulm Dis 2016; 3(4): 729-738.

11. Gorse GJ, O'Connor TZ, Young SL, et al. Impact of a winter respiratory virus season on patients with COPD and association with influenza vaccination. Chest 2006;130(4):1109-1116.

12. Seemungal T, Harper-Owen R, Bhowmik A, et al. Respiratory viruses, symptoms, and inflammatory markers in acute exacerbations and stable chronic obstructive pulmonary disease. Am J Respir Crit Care Med 2001;164(9):1618-1623. 